\section{2-D Spatial Smoothing for Multipath Coherent Signal Separation}

\author{
HONGYI WANG \\ Lucent Technologies
}

K. J. RAY LIU, Senior Member, IEEE

University of Maryland

The use of two-dimensional spatial smoothing (SS) to increase channel capacity of wireless communications system is discussed. The necessary and sufficient conditions on array configuration for applying SS to multiple signal classification (MUSIC), and adaptive beamforming algorithms are defined and proved. This array must have an orientational invariance structure with an ambiguity free center array, and the number of subarrays must be larger than or equal to the size of the largest group of coherent signals. We also studied the cause of ambiguities in a multipath environment. We found the necessary and sufficient conditions for a three-sensor array manifold to be ambiguity free and identified several higher order ambiguity situations. If an array is also central symmetric, the forward/backward SS (FBSS) can be used to improve the resolution. Finally, we extended our results to the estimation of signal parameters via rotational invariance techniques (ESPRIT). All the predicted results are verified by simulations.

Manuscript received June 14, 1995; revised April 20, 1996 and January 13, 1997.

IEEE Log No. T-AES/34/2/03186.

This work was supported in part by the NSF Grant MIP-9309506, the ONR Grant N00014-93-1-0566, and MIPS/Watkins Johnson.

Authors' current addresses: H. Wang, Network Wireless Systems, Lucent Technologies, Whippany, NJ 07981; K. J. R. Liu, Electrical Engineering Department and Institute for Systems Research, University of Maryland, College Park, MD 20742.

0018-9251/98/\$10.00 (C) 1998 IEEE

\section{INTRODUCTION}

As the digital signal processing technologies advance, the use of adaptive arrays to combat multipath fading and to reduce interference becomes increasingly valuable as a means of adding capacity to mobile communications.

There are many optimum adaptive array combining algorithms. Among them, the high resolution direction finding based constrained adaptive beamforming [1-3] and the reference signal based optimum spatial diversity array combining $[4,5]$ are considered most applicable in time division multiple access (TDMA) wireless communications and have attracted the most attention. In this work, we only consider the constrained adaptive beamforming approach. The key to making this technique effective in multipath fading environment is accurate direction of arrival (DOA) estimation of coherent or highly correlated signals.

The optimal weighted subspace fitting (WSF) DOA estimation [2] algorithm has been previously proposed to identify the coherent signals in a mobile communication environment. But the WSF and other optimal DOA estimation algorithms such as deterministic maximum likelihood (DML) [6] require optimization of a multidimensional nonlinear criterion function. Since this may be a difficult and time-consuming task, less expensive suboptimal approaches such as the multiple signal classification (MUSIC) algorithm [7] and estimation of signal parameters via rotational invariance techniques (ESPRIT) [8] are often employed. The MUSIC algorithm involves one-dimensional search, compared with multidimensional search inherent in the optimal approaches. However, computational efficient suboptimal DOA estimation techniques have the drawback of severe degradation of the estimation accuracy [12] in the presence of highly correlated or coherent signals. (Coherent signals also cause signal cancellation [13] in adaptive beamforming algorithms such as minimum-variance distortionless response (MVDR) and linearly constrained minimum-variance beamforming (LCMV) algorithms [9-11].) The multidimensional methods were still conceived to be more appropriate than the suboptimal approaches for coherent interference environment, despite their computational requirements.

The potential of adaptive array in wireless communication and the high computational requirements of the previously proposed optimal DOA estimation approaches motivate us to reevaluate a preprocessing scheme referred to as spatial smoothing (SS) which was proposed by Evans, et al. [14] and further developed by Shan, et al. [12, 15]. SS has been shown to be effective in decorrelating coherent signals. It thus makes suboptimal MUSIC algorithm and MVDR and LCMV beamforming algorithms effective in coherent interference environment. Such 
a scheme was only applied to uniformly spaced linear arrays. Later, Friedlander and Weiss [20] revealed that SS is not limited to uniform linear arrays. In [20] it stated that "the forward smoothing can be performed on any array which can be subdivided into subarrays which have the same configuration, but are shifted with respect to each other." However, they concluded the requirement is quite restrictive and therefore limits the types of arrays to which the backward/forward smoothing can be applied. They did not discuss the potential application of this technique in wireless communications where array with symmetric configurations are more relevant than arbitrary configurations (which includes both symmetric and nonsymmetric configurations). They developed SS with interpolated array $[20,21]$ technique to perform an approximate SS for an arbitrary array configuration.

The objective of this work is to further pursue SS, to decorrelate coherent signals using SS to achieve robustness in direction finding and to apply to mobile communications. We demonstrate that SS can be used on some two-dimensional arrays. By working on a smoothed data matrix obtained from SS, we can use MUSIC and ESPRIT effectively in a coherent interference environment and meanwhile achieve robustness in performance.

Specifically, we defined and proved the necessary and sufficient conditions on an array geometry for applying SS. They are: 1) such an array must have an orientational invariance structure, 2) its center array has an ambiguity-free array manifold, and 3) the number of subarrays is larger than or equal to the largest number of mutually coherent signals. We proved a sufficient condition for applying the forward/backward SS [23] (FBSS), which can further increase efficiency and estimation resolution. Finally, we extend the application of our results to ESPRIT.

The use of SS does not increase the computational complexity of either MUSIC, ESPRIT, or adaptive beamforming. Also, it can be used in conjunction with MUSIC or ESPRIT algorithm to provide an initialization for the WSF method to get a more accurate DOA estimation [19].

In many papers that dealt with DOA estimation with arrays of nonlinear geometry, ambiguity-free array manifolds were assumed. In [7] Schmidt discovered and defined the rank- $n$ ambiguity in an array manifold. In [26], Lo and Marple proved the conditions for a rank-2 ambiguity. In [24] ambiguities of linear arrays were studied. However, constructing a nonlinear array free of up to rank- $k$ ambiguities using only $(k+1)$ sensors remains a challenging problem [25]. In this work, we report a more thorough study on this issue. We proved the necessary and sufficient conditions for a three-sensor array manifold to be ambiguity free. We then identified several situations, for higher order sensor array manifolds, in which ambiguity may arise. Thus we get corresponding necessary conditions to design ambiguity-free center arrays and subarrays.

In Section II, we introduce MUSIC and SS. In Section III, we prove the necessary and sufficient conditions on two-dimensional array for applying SS, and consider the FBSS technique for applications in two-dimensional arrays. In Section IV, we study the cause of ambiguities in a multipath signal environment. In Section V, we present some practical considerations and simulation results. In Section VI, we expand our results to ESPRIT. Section VII concludes our work.

\section{ARRAY MODEL}

In this section, we briefly describe the array model for DOA estimation and beamforming.

The mathematical model is given as follows. Consider an array of $p$ sensors. Let $d$ narrowband plane waves $s_{1}(t), s_{2}(t), \ldots, s_{d}(t)$ impinge on the array at incident angles $\theta_{1}, \ldots, \theta_{d}$. There is also an additive white Gaussian noise vector $\mathbf{n}(t)$, where $\mathbf{n}(t)=\left[n_{1}(t), \ldots, n_{p}(t)\right]^{T}$, and $n_{i}(t), i=1, \ldots, p$ have zero mean and variance $\sigma^{2}$. The noise received by any sensor is assumed to be uncorrelated with signals and with noise received by any other sensors. The received signals of the array can be expressed as

$$
\mathbf{r}(t)=A \mathbf{s}(t)+\mathbf{n}(t)
$$

where $\mathbf{r}(t)=\left[r_{1}(t), \ldots, r_{p}(t)\right]^{T}$, and $r_{i}(t)$ is the received signal at the $i$ th sensor, and $A$ is a $p \times d$ matrix, $p>d$,

$$
A=\left[\mathbf{a}\left(\theta_{1}\right), \ldots, \mathbf{a}\left(\theta_{d}\right)\right]
$$

where $\mathbf{a}\left(\theta_{i}\right)$ is the steering vector associated with the arrival angle $\theta_{i}$.

The array output covariance matrix has the form:

$$
R=E\left(\mathbf{r}(t) \mathbf{r}^{H}(t)\right)=A R_{s} A^{H}+\sigma^{2} I
$$

where $R_{s}=E\left(\mathbf{s}(t) \mathbf{s}^{H}(t)\right)$. Let $\left\{\lambda_{1} \geq \lambda_{2} \geq \cdots \geq \lambda_{p}\right\}$ and $\left\{\nu_{1}, \nu_{2}, \ldots, \nu_{p}\right\}$ denote the eigenvalues and corresponding eigenvectors of $R$. When the $d$ incoming signals are noncoherent, and the matrix $A$ is of full column rank, the MUSIC algorithm can be used to estimate the angles of the incoming signals as the peaks of the MUSIC estimates

$$
\hat{S}_{\mathrm{MUSIC}}(\theta)=\frac{1}{\sum_{l=d+1}^{p}\left|\mathbf{a}(\theta)^{H} \nu_{l}\right|^{2}} .
$$

However, when the signals are coherent, $R_{s}$ is then singular. The MUSIC algorithm is no longer applicable. In the case of a uniformly spaced linear array, with a sensor spacing $\Delta$, the SS [12] algorithm can be applied to achieve the nonsingularity of the modified covariance matrix of the signals. This technique begins by dividing a uniform linear array with $L$ sensors into $K$ overlapping subarrays of size 
$p$, with sensors $\{1, \ldots, p\}$ forming the first subarray, sensors $\{2, \ldots, p+1\}$ forming the second subarray, etc. The spatially smoothed covariance matrix is defined as the average of the subarray covariances:

$$
\bar{R}=\frac{1}{K} \sum_{k=1}^{K} R_{k}=A_{1} \bar{R}_{s} A_{1}^{H}+\sigma^{2} I
$$

where $R_{k}$ is the covariance matrix associated with the $k$ th subarray, $\bar{R}_{s}$ is the modified covariance matrix of the signals, and has been proved [12] to be full rank when $K \geq d, A_{1}$ is a $p \times d$ matrix consisting of steering vectors associated with the first subarray, The signals are thus progressively decorrelated [16]. However, linear arrays have limitations in the domain of estimable DOAs. It has been shown in [27] that $\bar{R}_{s}$ can be decomposecl as follows:

$$
\bar{R}_{s}=\frac{1}{K} C C^{H}
$$

where $C=P A^{T}$ with $P=\operatorname{diag}\left(p_{1}, p_{2}, \ldots, p_{d}\right)$, and other. But only sufficient conditions were provided. In this section, we prove necessary and sufficient conditions on array geometries for implementing the general SS.

First, we give the following lemmas.

LEMMA 1 For steering matrices $A$ and $B$, given by $A=\left[\mathbf{a}\left(\theta_{1}\right), \ldots, \mathbf{a}\left(\theta_{d}\right)\right]$ and $B=\left[\mathbf{b}\left(\theta_{1}\right), \ldots, \mathbf{b}\left(\theta_{d}\right)\right]$, there exists a mapping relation $B=A C$ if and only if $C$ is a diagonal matrix.

LEMMA 2 For $K$ steering matrices $A_{1}, A_{2}, \ldots, A_{K}$, each $A_{i}$ can be mapped to a steering matrix $B$ if and only if there exists a mapping relation $A_{j}=A_{i} C_{i j}$ for any $i$ and $j$.

The proofs of both lemmas are given in the Appendix.

Consider an array that is divided into $K$ subarrays. Suppose $A_{i}$ and $A_{j}$ are the steering matrices associated with the $i$ th and the $j$ th subarrays, and there are $d$ signals with incoming angles $\theta_{1}, \ldots, \theta_{d} . A_{i}$

$$
A=\left[\begin{array}{cccc}
1 & \cdots & 1 \\
\exp \left(-j 2 \pi \frac{\Delta}{\lambda} \sin \left(\theta_{1}\right)\right) & \exp \left(-j 2 \pi \frac{\Delta}{\lambda} \sin \left(\theta_{2}\right)\right) & \cdots & \exp \left(-j 2 \pi \frac{\Delta}{\lambda} \sin \left(\theta_{d}\right)\right) \\
\exp \left(-j 4 \pi \frac{\Delta}{\lambda} \sin \left(\theta_{1}\right)\right) & \exp \left(-j 4 \pi \frac{\Delta}{\lambda} \sin \left(\theta_{2}\right)\right) & \cdots & \exp \left(-j 4 \pi \frac{\Delta}{\lambda} \sin \left(\theta_{d}\right)\right) \\
\vdots & \vdots & \ddots & \vdots \\
\exp \left(-j 2 K \pi \frac{\Delta}{\lambda} \sin \left(\theta_{1}\right)\right) & \exp \left(-j 2 K \pi \frac{\Delta}{\lambda} \sin \left(\theta_{2}\right)\right) & \cdots & \exp \left(-j 2 K \pi \frac{\Delta}{\lambda} \sin \left(\theta_{d}\right)\right)
\end{array}\right]
$$

When incoming signals are closely spaced, the columns of both $A$ and $A_{1}$ become almost linearly dependent [27]. The dependency increases drastically when some of $\sin \left(\theta_{i}\right), i=1, \ldots, d$ approach 1 for DOAs near $90^{\circ}$. As a result, the performance of a linear array deteriorates quickly when some DOAs approach $90^{\circ}$. The lack of performance robustness of a linear array is even more severe when SS technique is applied, because in the smoothed covariance matrix, not only the steering matrix $A_{1}$, but also $A$ is ill-conditioned in the situation described above. A general SS technique that is robust and can be applied to directionally independent arrays is thus more desirable.

\section{TWO-DIMENSIONAL SS}

\section{A. Orientational Invariance Structure}

According to [20], in general, forward smoothing can be performed on an array which can be subdivided into stibarrays which have the same configuration, but are shifted with respect to each can be written as

$$
A_{i}=\left[\mathbf{a}_{i}\left(\theta_{1}\right), \mathbf{a}_{i}\left(\theta_{2}\right), \ldots, \mathbf{a}_{i}\left(\theta_{d}\right)\right]
$$

where $\mathbf{a}_{i}^{T}\left(\theta_{k}\right)=\left[e^{-j \phi_{i 1}\left(\theta_{k}\right)}, e^{-j \phi_{i 2}\left(\theta_{k}\right)}, \ldots, e^{-j \phi_{i p}\left(\theta_{k}\right)}\right], k=$ $1, \ldots, d$, is the steering vector associated with the $i$ th subarray, and $\phi_{i l}\left(\theta_{k}\right), l \in\{1, \ldots, p\}$, is the phase delay of the $k$ th signal at the $l$ th sensor of the $i$ th subarray from the first sensor of the first subarray. We refer to the sensor of an array associated with the $l$ th row of a steering matrix of the array as the $l$ th sensor of the array.

Let $\Delta_{i j l}, 1 \leq l \leq p$, represent the distance between the $l$ th sensor in the $i$ th subarray and the $l$ th sensor in the $j$ th subarray. Let $\beta_{i j l}$ represent the angle of the line on which these two sensors are located. If the $i$ th and the $j$ th subarrays are identical and have the same orientation, i.e., all $\Delta_{i j l}$ for $l=1, \ldots, p$ are equal and all $\beta_{i j l}, l=1, \ldots, p$ are equal, then the phase delay of a signal with an incoming angle $\theta_{k}$ from each sensor in the $i$ th subarray to the corresponding sensor in the $j$ th subarray is the same according to the far field assumption. We denote this phase delay by $\Phi_{i j}\left(\theta_{k}\right)$. 
For any $l \in\{1, \ldots, p\}$, we have

$$
\Phi_{i j}\left(\theta_{k}\right)=\phi_{j l}\left(\theta_{k}\right)-\phi_{i l}\left(\theta_{k}\right)=2 \pi \frac{\Delta_{i j l}}{\lambda} \sin \left(\beta_{i j l}-\theta_{k}+\frac{\pi}{2}\right)
$$

then $A_{j}=A_{i} C_{i j}$, where $C_{i j}$ is a diagonal matrix with the $m$ th diagonal element $\exp \left(-j \Phi_{i j}\left(\theta_{m}\right)\right)$. The identical and orientational invariance properties between two subarrays guarantee a mapping relation between their steering matrices.

On the other hand, if $A_{j}=A_{i} C$, by Lemma 1,C should be a diagonal matrix and can be represented by $C=\operatorname{diag}\left\{c_{11}\left(\theta_{1}\right), c_{22}\left(\theta_{2}\right), \ldots, c_{d d}\left(\theta_{d}\right)\right\}$. It requires that

$$
\begin{aligned}
& \exp \left(-j \phi_{i l}\left(\theta_{k}\right)\right) c_{k k}\left(\theta_{k}\right) \\
& =\exp \left(-j \phi_{j l}\left(\theta_{k}\right)\right) \quad \text { for } \quad l=1, \ldots, p
\end{aligned}
$$

which can be simplified to

$$
\phi_{j l}\left(\theta_{k}\right)-\phi_{i l}\left(\theta_{k}\right)=\Phi_{i j}^{\prime}\left(\theta_{k}\right)+2 \pi n, \quad \text { for } \quad l=1, \ldots, p
$$

where $n$ can be any integer. The relation in (11) holds for all $\theta_{k}$ in $[0,360)$ only if $\Delta_{i j 1}=\Delta_{i j 2}=\cdots=$ $\Delta_{i j p}$ and $\beta_{i j 1}=\beta_{i j 2}=\cdots=\beta_{i j p}$, i.e., the $i$ th and the $j$ th subarrays must be identical and have the same orientation. Thus, we have Lemma 3.

LEMMA 3 Suppose $A_{i}$ and $A_{j}$ are steering matrices associated with the ith and the $j$ th subarrays. The sensors in each subarray are numbered in the same sequence. There exists a mapping relation $A_{j}=A_{i} C_{i j}$ if and only if the ith and the $j$ th subarrays are identical and have the same orientation.

From Lemmas 2 and 3, we have the following theorem.

THEOREM 1 Suppose an array can be divided into $K$ subarrays, each having a $p \times d$ steering matrix $A_{i}$, $i=1,2, \ldots, K$. All $A_{1}, A_{2}, \ldots, A_{K}$ can be mapped to a $p \times d$ steering matrix $B$ by $A_{i}=B D_{i}$ if and only if all these subarrays are identical and have the same orientation.

We call the array structure held by an array satisfying condition in Theorem 1 the orientational invariance structure. A more rigorous definition is given as follows.

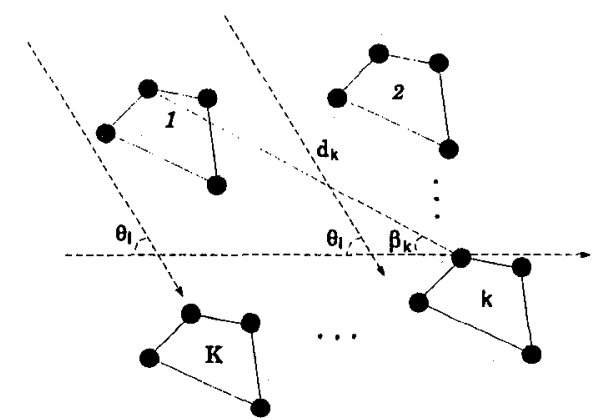

Fig. 1. Orientational Invariance sensor array geometry.

DEFINITION 1 (Orientational Invariance Structure). An array has an orientational invariance structure if it can be divided into subarrays that are identical and have the same orientation.

For an array with orientational invariance structure, we can consider each subarray as one element located at its first sensor. Then all these elements form a center array. A more rigorous definition for center array is given as follows:

DEFINITION 2 (Center Array). If an array with orientational invariance structure is divided into subarrays (which can have overlap), then the collection of all the first sensors of these subarrays form a center array.

\section{B. Necessary and Sufficient Conditions}

Suppose an array has an orientational invariance structure. Moreover, its center array has an ambiguity-free structure and the number of subarrays is larger than or equal to the largest number of mutually coherent signals. The $p \times d$ steering matrices $A_{1}, A_{2}, \ldots, A_{K}$ are associated with the subarrays $1,2, \ldots, K$, respectively, and $d_{k}$ is the distance between the first sensor in the first subarray and the first sensor in the $k$ th subarray. The angle $\beta_{k}$ represents the direction of the line on which the first sensor in the first subarray and the first sensor in the $k$ th subarray are located (see Fig. 1). We have

$$
A_{k}=A_{1} D_{k}, \quad k=2, \ldots, K
$$

where

$$
D_{k}=\left[\begin{array}{lll}
\exp \left(-j \frac{2 \pi d_{k}}{\lambda} \sin \left(\beta_{k}-\theta_{1}+\frac{\pi}{2}\right)\right) & & \\
& \exp \left(-j \frac{2 \pi d_{k}}{\lambda} \sin \left(\beta_{k}-\theta_{2}+\frac{\pi}{2}\right)\right) & \\
& \ddots & \exp \left(-j \frac{2 \pi d_{k}}{\lambda} \sin \left(\beta_{k}-\theta_{d}+\frac{\pi}{2}\right)\right)
\end{array}\right] .
$$


The covariance matrix of the $k$ th subarray is thus given by

$$
R_{k}=A_{1} D_{k} R_{s} D_{k}^{H} A_{1}^{H}+\sigma^{2} I
$$

where $R_{s}$ is the covariance matrix of the source. The spatially smoothed covariance matrix is defined as the average of the subarray covariances

$$
\bar{R}=\frac{1}{K} \sum_{k=1}^{K} R_{k}=A_{1} \bar{R}_{s} A_{1}+\sigma^{2} I
$$

where $\bar{R}_{s}$ is the modified covariance matrix of the signal given by

$$
\bar{R}_{s}=\frac{1}{K} \sum_{k=1}^{K} D_{k} R_{s} D_{k}^{H} .
$$

We show in the following that $\bar{R}_{s}$ is nonsingular. First, $\bar{R}_{s}$ can be written als
Let $C$ denote the Hermitian square root of $(1 / K) R_{s}$, i.e.,

$$
C C^{H}=\frac{1}{K} R_{s} .
$$

It follows that

$$
\bar{R}_{s}=G G^{H}
$$

where $G$ is a $d \times K d$ block matrix given by

$$
G=\left[\begin{array}{llll}
C & D_{2} C & \cdots & D_{K} C
\end{array}\right] .
$$

Clearly, the rank of $\bar{R}_{s}$ is equal to the rank of $G$.

Suppose there are $q$ groups of signals in $d$ incoming signals, with $l_{i}, i=1, \ldots, q$, correlated signals in each group. $R_{s}$ must be a block diagonal matrix with block size $l_{i}, i=1, \ldots, q$. We can thus get a corresponding block diagonal matrix $C$. If we exchange the columns of a matrix, the rank of the matrix does not change. By grouping columns of similar elements, we can verify that

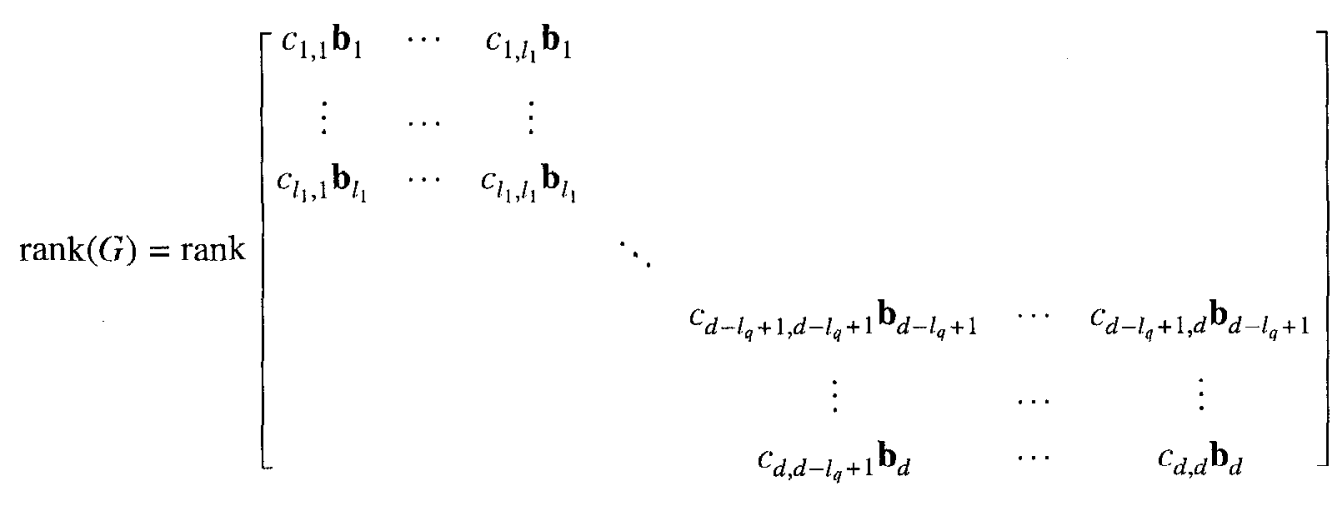

$c_{i j}$ is the $i j$ th element of matrix $C$, and $\mathbf{b}_{i}(i=1, \ldots, d)$ is the $1 \times K$ row vector given by

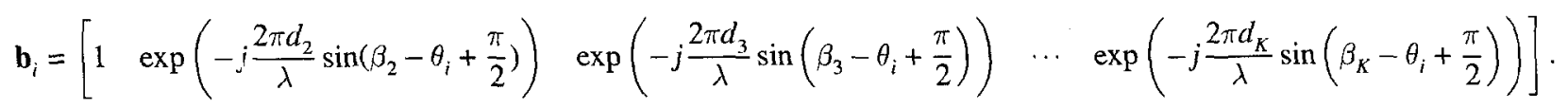

$$
\begin{aligned}
& \bar{R}_{s}= \\
& {\left[\begin{array}{llll}
I & D_{2} & \cdots & D_{K}
\end{array}\right]\left[\begin{array}{cccc}
\frac{1}{K} R_{s} & & & \\
& \frac{1}{K} R_{s} & & \\
& & \ddots & \\
& & & \frac{1}{K} R_{s}
\end{array}\right]\left[\begin{array}{c}
I \\
D_{2}^{H} \\
\vdots \\
D_{K}^{H}
\end{array}\right] .}
\end{aligned}
$$

Each row of matrix $C$ has at least one non-zero element because the energy of each signal is non-zero. It is observed that $\mathbf{b}_{i}$ is the transpose of the steering vector associated with the center array. Since the center array is assumed to have ambiguity free array manifold, when $K \geq \max \left\{l_{1}, l_{2}, \ldots, l_{q}\right\}$, all the $\mathbf{b}$ vectors associated with all the signals within a group of coherent signals are thus linearly independent. Therefore, $G$ is of full row rank and the modified covariance matrix $\bar{R}_{s}$ is of full rank. Otherwise, if $K<\max \left\{l_{1}, l_{2}, \ldots, l_{q}\right\}$, we will see that $\bar{R}_{s}$ is rank 
deficient. We assume that $R_{s i}$ is the correlation matrix associated with the ith group of coherent signals. Thus, $R_{s i}$ has rank 1 and can be expressed as $\lambda_{i} \mathbf{h}_{i} \mathbf{h}_{i}^{H}$ where $\lambda_{i}$ and $\mathbf{h}_{i}$ are the corresponding eigenvalue and eigenvector of $R_{s i}$. We have

$$
\begin{aligned}
R_{s} & =\left[\begin{array}{llll}
R_{s 1} & & & \\
& R_{s 2} & & \\
& \ddots & \\
& & & R_{s q}
\end{array}\right] \\
& =\left[\begin{array}{llll}
\lambda_{1} \mathbf{h}_{1} \mathbf{h}_{1}^{H} & & & \\
& \lambda_{2} \mathbf{h}_{2} \mathbf{h}_{2}^{H} & & \\
& & \ddots & \\
& & & \lambda_{q} \mathbf{h}_{q} \mathbf{h}_{q}^{H}
\end{array}\right]
\end{aligned}
$$

and
C. Further Improvement

To get a smoothed nonsingular covariance matrix $\bar{R}_{s}$ by using the SS technique, we need $K \geq \max \left\{l_{1}, l_{2}, \ldots, l_{q}\right\}$. We can further reduce the number of subarrays by getting another $K$ backward subarrays similar to the case in a linear array [23]. Although, the FBSS [23] can always be applied in a uniformly spaced linear array. For two-dimensional array, there is some requirements on the geometry for successful implementation of the backward method. The necessary condition [20] is that each subarray must have center symmetry. The sufficient condition we found is that the whole array is center symmetry in addition to its configuration conditions for applying SS.

If an array is central symmetric, we can get $K$ additional backward subarrays by reversing the order of the subarrays and the order of the sensors within

$$
\bar{R}_{s}=\left[\begin{array}{llll}
\sum_{k=1}^{K} \lambda_{1}\left(D_{k_{1}} \mathbf{h}_{1}\right)\left(D_{k_{1}} \mathbf{h}_{1}\right)^{H} & & \\
& \sum_{k=1}^{K} \lambda_{2}\left(D_{k_{2}} \mathbf{h}_{2}\right)\left(D_{k_{2}} \mathbf{h}_{2}\right)^{H} & & \\
& \ddots & \\
& & \sum_{k=1}^{K} \lambda_{q}\left(D_{k_{q}} \mathbf{h}_{q}\right)\left(D_{k_{q}} \mathbf{h}_{q}\right)^{H}
\end{array}\right]
$$

where $D_{k_{i}}$ is a diagonal matrix consisting of $l_{i}$ diagonal elements of $D_{k}$ which are associated with all the DOAs from $i$ th group of coherent signals. Since

$$
\operatorname{rank}\left(\sum_{k=1}^{K} \lambda_{i}\left(D_{k_{i}} \mathbf{h}_{i}\right)\left(D_{k_{i}} \mathbf{h}_{i}\right)^{H}\right) \leq \min \left(K, l_{i}\right)
$$

and

$$
\operatorname{dim}\left(\sum_{k=1}^{K} \lambda_{i}\left(D_{k_{i}} \mathbf{h}_{i}\right)\left(D_{k_{i}} \mathbf{h}_{i}\right)^{H}\right)=l_{i} \times l_{i}
$$

thus $\bar{R}_{s}$ is rank deficient if $K \leq \max \left(l_{1}, l_{2}, \ldots, l_{q}\right)$.

If the center array is not ambiguity free, then all the $\mathbf{b}$ vectors associated with all the signals within a group of coherent signals can be linearly dependent, $G$ cannot be ensured to be of full row rank, and neither can $R_{s}$.

From Theorem 1 and the proof above, we get the following theorem.

THEOREM 2 SS can be applied to a two-dimensional array to obtain a full rank smoothed signal covariance matrix if and only if an array has an orientational invariance structure, its center array has an ambiguity-free structure, and the number of subarrays is larger than or equal to the size of the largest group of coherent signals. each subarray. Let $\mathbf{r}_{k}^{b}(t)$ denote the complex conjugate of the output of the $k$ th backward subarray for $k=$ $1, \ldots, K$. We have

$$
\mathbf{r}_{k}^{b}(t)=A_{1} D_{k}\left(D_{L} \mathbf{s}(t)\right)^{*}+\tilde{\mathbf{n}}^{*}(t)
$$

where $\tilde{\mathbf{n}}(t)$ is an additive white Gaussian noise vector, $D_{L}$ is a diagonal matrix with the $i$ th diagonal element given by $\exp \left(-j 2 \pi\left(d_{K p} / \lambda\right) \sin \left(\beta_{K p}-\theta_{i}+(\pi / 2)\right)\right)$ and $d_{K p}$ is the distance between the first sensor in the first forward subarray and the first sensor in the first backward subarray. The angle $\beta_{K p}$ represents the direction of the line on which the two sensors are located.

The covariance matrix of the $k$ th backward subarray is given by

$$
R_{k}^{b}=A_{1} D_{k} R_{s}^{b} D_{k}^{H} A_{1}^{H}+\sigma^{2} I
$$

with

$$
R_{s}^{b}=E\left(D_{L}^{*} \mathbf{s}^{*}(t) \mathbf{s}^{T}(t) D_{L}^{T}\right)=D_{L}^{*} R_{s}^{*} D_{L}^{T} .
$$

Define the spatially smoothed backward subarray covariance matrix $\bar{R}^{b}$ as the average of these subarray covariance matrices, i.e.,

$$
\bar{R}^{b}=\frac{1}{K} \sum_{k=1}^{K} R_{k}^{b}=A_{1} \bar{R}_{s}^{b} A_{1}^{H}+\sigma^{2} I
$$


where

$$
\bar{R}_{\mathrm{s}}^{b}=\frac{1}{K} \sum_{k=1}^{K} D_{k} R_{s}^{b} D_{k}^{H}
$$

and define the forvard/backward smoothed covariance matrix $\tilde{R}$ as the average of $\bar{R}$ in (15) and $\bar{R}^{b}$, i.e.,

$$
\tilde{R}=\frac{\bar{E}^{i}+\bar{R}^{b}}{2}=A_{1} \tilde{R}_{s} A_{1}^{H}+\sigma^{2} I .
$$

It follows that

$$
\tilde{R}_{s}=\frac{\bar{R}_{s}+\bar{R}_{s}^{b}}{2} .
$$

We can show, in a similar way as in the case of a linear array [23], that the modified source covariance matrix $\tilde{R}_{s}$ is nonsingular as long as $2 K \geq$ $\max \left\{l_{1}, l_{2}, \ldots, l_{q}\right\}$.

\section{AMBIGUITY-FREE ARRAY STRUCTURE}

To perform SS, we need an ambiguity-free center array manifold. Also, to perform MUSIC, we further require ambiguity free subarray manifolds. Ambiguity arises when a steering vector can be expressed as a linear combination of other steering vectors in an array manifold [7]. For a uniformly spaced linear array, rank-1 ambiguity [7] cannot be avoided since the DOAs which are "mirror images" with respect to the array line, have the same steering vector. This limits the range of DOAs estimable by a uniformly spaced linear array to within $180^{\circ}$. Suppose an array has $p$ elements, then rank- $p$ [7] ambiguities cannot be avoided. In this paper, an ambiguity free array manifold of an array of $p$ sensors refers here to rank- $(p-1)$ ambiguity free. Generally, to avoid ambiguity, an array used for high-resolution DOA estimation must have a proper structure. An ambiguity-free array manifold has been assumed in several papers $[8,17,19]$. Our attempt is to identify all the situations in which ambiguity may arise. One of our guidelines in designing arrays is to avoid these identified ambiguities.

THEOREM 3 In an azimuth-only system, the necessary and sufficient condition for an ambiguity-free three-sensor array manifold is that all these three sensors are not on one line and that the distance between any two sensors is less than or equal to $\lambda / 2$.

The proof is given in the Appendix (see Fig. 2).

We can see in general that 1) rank-1 ambiguity occurs not only in uniformly spaced linear arrays but also in rectangular arrays with sensors having a uniform spacing of $\lambda / 2$ along either $x$-axis or $y$-axis, 2) rank-2 ambiguity occurs in an array that consists of two parallel linear: arrays with an identical uniform sensor spacing that is larger than $\lambda / 2,3$ ) rank-3 ambiguity occurs in an array that consists of three

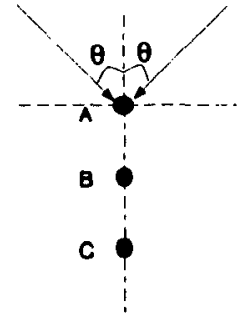

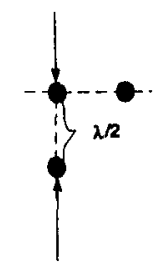

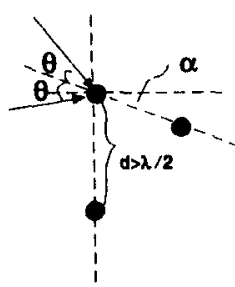

(a)

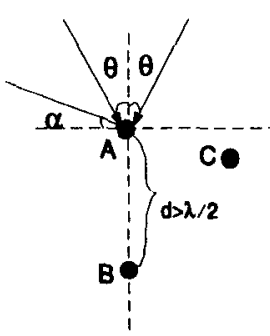

(b)
Fig. 2. Three-sensor array structures that can cause ambiguities.

parallel linear arrays with an identical uniform sensors spacing that is larger than $\lambda / 2$, and 4) higher order ambiguity occurs if more than $\lceil k / 2\rceil$ sensors are on one line in a $k$ sensor array or if an array consists of $m$ parallel linear arrays with an identical uniform sensor spacing that is larger than $\lambda / 2\lfloor m / 2\rfloor$. These situations are shown schematically in Fig. 3(a)-(d). In Fig. 3(b) and (c), the angles $\theta$ and $\alpha$ satisfy the following constraint:

$$
2 \pi \frac{d}{\lambda} \sin (\alpha)+2 k \pi=2 \pi \frac{d}{\lambda} \sin (\theta), \quad k \in\{1,2, \ldots\} .
$$

In Fig. 3(d), the angles $\theta, \beta$, and $\alpha$ satisfy the following constraint:

$$
2 \pi \frac{d}{\lambda} \sin (\alpha)+2 k_{1} \pi=2 \pi \frac{d}{\lambda} \sin (\beta)+2 k_{2} \pi=2 \pi \frac{d}{\lambda} \sin (\theta)
$$

where $k_{1}, k_{2} \in\{1,2, \ldots\}$ and $k_{1} \neq k_{2}$.

To get an ambiguity-free array manifold, it is necessary to avoid these identified situations.

\section{IMPLEMENTATION AND SIMULATION RESULTS}

\section{A. Some Practical Considerations}

To determine the source coherency structure, we can use smoothed rank profile (SRP) [28]. On the other hand, we can estimate the maximum number of incoming angles according to the multipath environment. In this work, limited by space, we assume the number of incoming signals is given.

In practice, we can perform FBSS by setting up a special data matrix. Specifically, for the $n$th snapshot 

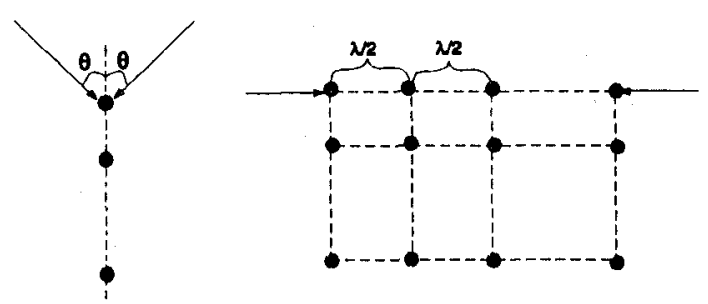

(a)



(b)

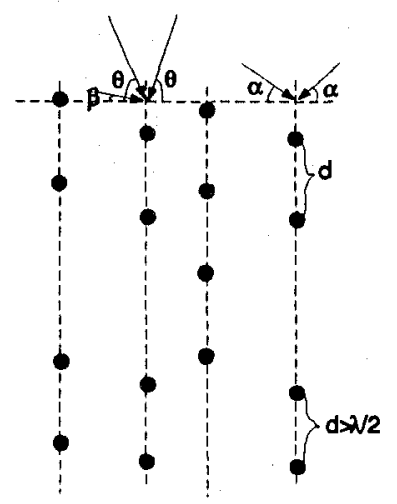

(d)

Fig. 3. High-order array structures that can cause ambiguities. (a) Rank-1 ambiguity. (b) Rank-2 ambiguity. (c) Rank-3 ambiguity. (d) High-order ambiguity.

we set up the data matrix

$A^{H}(n)=$
$\left[\begin{array}{cccccc}u(p, 1, n) & \cdots & u(p, K, n) & u^{*}(1, K, n) & \cdots & u^{*}(\mathbf{1}, 1, n) \\ u(p-1,1, n) & \cdots & u(p-1, K, n) & u^{*}(2, K, n) & \cdots & u^{*}(2,1, n) \\ \vdots & \ddots & \vdots & \vdots & \ddots & \vdots \\ u(1,1, n) & \cdots & u(1, K, n) & u^{*}(p, K, n) & \cdots & u^{*}(p, 1, n)\end{array}\right]$

where $u(i, j, n)$ denotes the sample taken at the $i$ th sensor of the $j$ th subarray. For the totality of $N$ snapshots, we can define the overall data matrix

$$
A^{H}=\left[A^{H}(1), A^{H}(2), \ldots, A^{H}(N)\right] .
$$

It follows that the averaged smoothed correlation matrix $\tilde{R}$ (as defined in (32)) can be estimated as follows:

$$
\tilde{R}=\frac{1}{2 N K} A^{H} A .
$$

As we know, more robust results can be obtained from data domain rather than from covariance domain [22]. We can proceed with MUSIC [22] algorithm or MVDR [10, 22] beamforming algorithm based on $A$ instead of $\tilde{R}$.

An array needs to be chosen for applying SS. Such an array should satisfy all conditions aforementioned. An omnidirectional circular array has been a conventional choice for mobile communications [1,2], and there have been active research efforts to find a preprocessing scheme for the circular array to handle the coherent interference [29]. However, we can see clearly from our discussion that a single circular array is not orientational invariant. Therefore it does not satisfy the necessary condition for applying SS. This implies that the circular array cannot overcome the coherent interference by using the SS technique. For some circular arrays with central symmetric, we can apply FBSS to handle two coherent signals. To handle more than two coherent signals, several parallel circular arrays have to be used.

\section{B. Simulation Results}

In this section, we present some simulation results on MUSIC algorithm to show the applications and effectiveness of our SS and FBSS. We choose a square array, which has an orientational invariance structure, central symmetric, and a sensor spacing less than $\lambda / 2$.

EXAMPLE 1 In a fading wireless channel, coherent signals are inevitable. Fig. 4 shows a typical example of the distribution of scatters in a mobile radio environment [31]. We can treat all the reflected signals from local scatterers as coming from a super position. Those reflected signals from far away scatterers such as high rise buildings or mountains are from another super position. Each group of local scatterers spans a small angle with respect to the base station. there are many reflected signals within each small angle spread. These angles cannot be resolved even with high resolution MUSIC algorithm. They will be treated as one signal that suffers from flat fading. Thus the necessary angles to be estimated is seven in Fig. 4. There are two groups of coherent fading signals with three and two coherent signals in each. The others are noncoherent fading signals.

We use a dense square array of sixteen sensors as the base station antenna. The array contains 4 subarrays each of 9 sensors. The spacing between two neighboring sensors is $0.45 \lambda$. The wireless communication environment in Fig. 4 is simulated. The array receives signals from four mobile users. All of them move at $60 \mathrm{mi} / \mathrm{h}$. The carrier frequency 


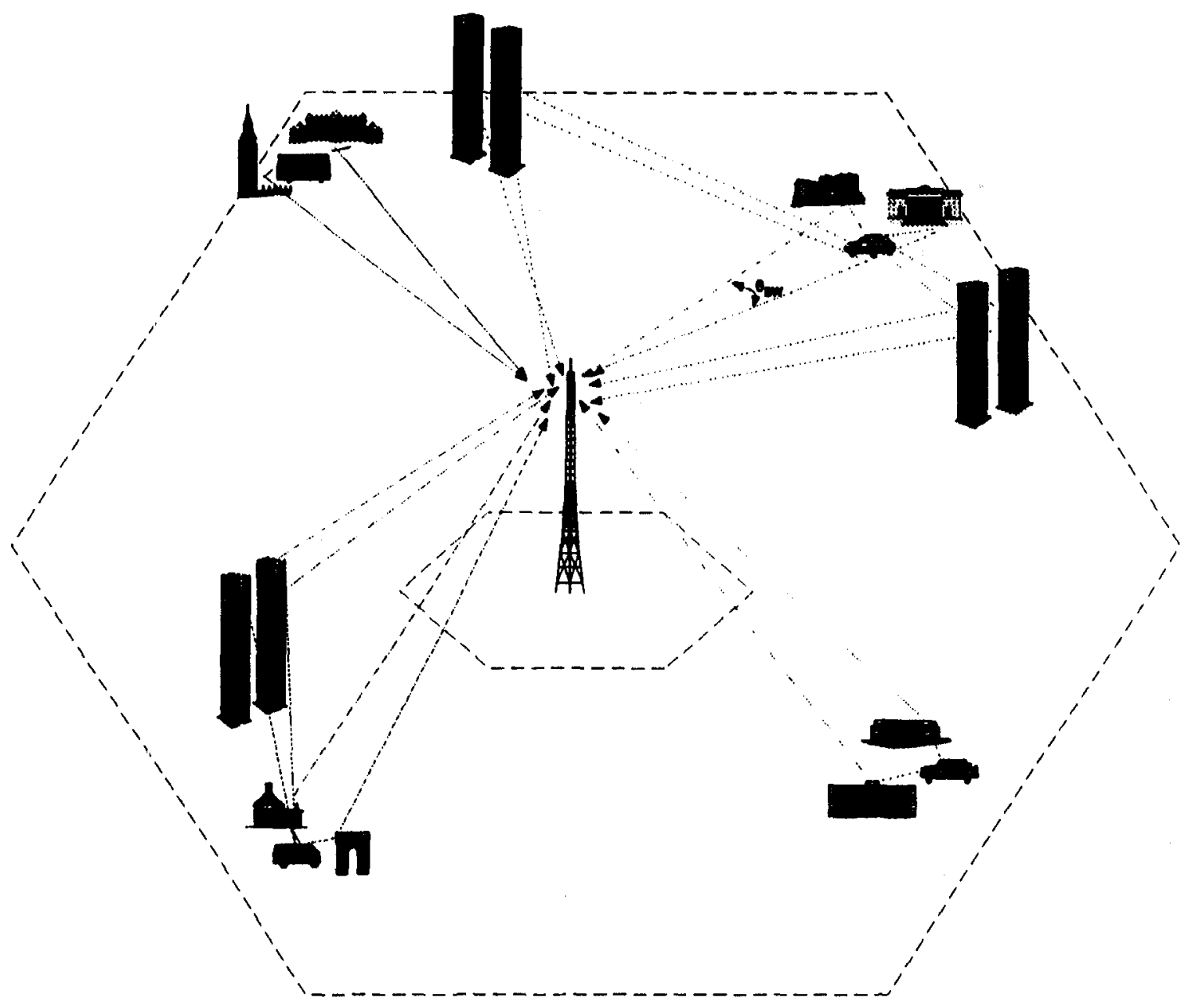

Fig. 4. Multiuser frequency selective channel.

is $900 \mathrm{MHz}$. The modulated data rate is $24.3 \mathrm{ksym} / \mathrm{s}$, which is the same as in IS-136 standard (an Electronic Industry Association Interim Standard). The maximum Doppler frequency $f d_{\max }=80 \mathrm{~Hz}$. There are 40 reflected signals from each local scatterer or each distant scatterer. These signals uniformly span a $4^{\circ}$ angle. The combined signals-to-noise ratio is $10 \mathrm{~dB}$.

The received array data is given as

$$
\mathbf{r}(t)=A * \mathbf{s}(t)+\mathbf{n}(t)
$$

where the steering matrix $A=\left[A_{1}, A_{2}, \ldots, A_{7}\right] ; A_{i}=$ $\left[a_{i 1}, a_{i 2}, \ldots, a_{i 40}\right]$

$$
a_{i j}=\left(\begin{array}{c}
\exp \left(-j 2 \pi\left(f_{o}+f_{d}\right) \cdot \frac{0.45 \lambda}{c}\right) \\
\vdots \\
\exp \left(-j 2 \pi\left(f_{o}+f_{d}\right) \cdot \frac{0.45 \lambda}{c} \cdot\left(3 \cdot \cos \left(\theta_{i, j}\right)\right)\right. \\
\vdots \\
\exp \left(-j 2 \pi\left(f_{o}+f_{d}\right) \cdot \frac{0.45 \lambda}{c} \cdot\left(3 \cdot \sin \left(\theta_{i, j}\right)\right)\right) \\
\vdots \\
\exp \left(-j 2 \pi\left(f_{o}+f_{d}\right) \cdot \frac{0.45 \lambda}{c} \cdot\left(3 \cdot \cos \left(\theta_{i, j}\right)+3 \cdot \sin \left(\theta_{i, j}\right)\right)\right)
\end{array}\right)
$$

is a $16 \times 1$ steering vector

$$
\begin{aligned}
\mathbf{s}(t)= & {\left[s_{1,1}(t), \ldots, s_{1,40}(t), s_{2,1}(t), \ldots,\right.} \\
& \left.s_{2,40}(t), \ldots, s_{7,1}(t), \ldots, s_{7,40}(t)\right]^{H}
\end{aligned}
$$

is a signal vector, $s_{n, k}(t)$ is the $k$ th reflected signal from the $n$th group of scatterers

$$
s_{n, k}(t)=\frac{1}{40} \exp \left(j 2 \pi\left(f d_{n, k} \cdot t+\phi_{n, k}\right)\right)
$$

The phase distortion $\phi_{n, k}$ is uniformly distributed in $[0,2 \pi]$. The Doppler frequency $f d_{n, k}=f d_{\max } \times$ $\cos (2 \pi(k-1) / 40) . k=1, \ldots, 40$

$$
\mathbf{n}(t)=\left[n_{1}(t), \ldots, n_{16}(t)\right]^{H}
$$

is a noise vector.

Reflected signal for user 1 is from $10^{\circ}$ to $14^{\circ}, 20^{\circ}$ to $24^{\circ}$ and $100^{\circ}$ to $104^{\circ}$. Reflected signal for user 2 is from $120^{\circ}$ to $124^{\circ}$. Reflected signal for user 3 is from $200^{\circ}$ to $204^{\circ}$ and $220^{\circ}$ to $224^{\circ}$. Reflected signal for user 4 is from $300^{\circ}$ to $304^{\circ}$.

A total of 162 samples are used. We apply FBSS first and then MUSIC. Simulation results are shown in Fig. 5. According to the DOAs information, we can 


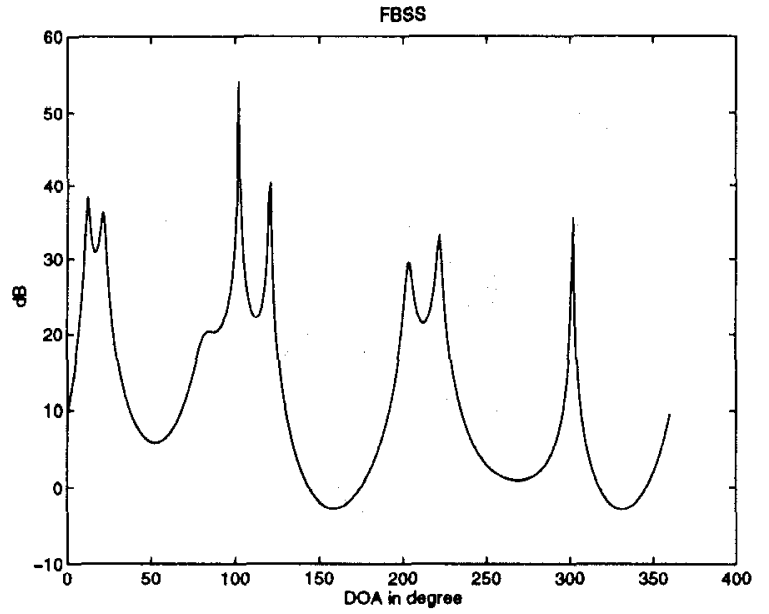

Fig. 5. DOA estimation of four groups of coherent signals at $\left(10^{\circ}, 20^{\circ}, 100^{\circ}\right),\left(120^{\circ}\right),\left(200^{\circ}, 220^{\circ}\right),\left(300^{\circ}\right)$ based on sixteen-sensor square array.

further perform constrained beamforming and thus can achieve spatial division multiple access (SDMA) [32] in a multipath environment.

EXAMPLE 2 In the previous examples, we demonstrated the effectiveness of SS using square arrays. Square arrays are efficient in terms of sensor reuse rate. One can also use other kinds of planar arrays which satisfy the necessary and sufficient conditions stated in Section III.

An alternative way of using planar arrays to cover all the azimuth angles is to use two crossed uniformly spaced linear arrays. Spatial smoothing and DOAs estimation can be done on each linear array. Ambiguities related to linear arrays can be largely avoided by searching for common estimated DOAs from both arrays. However we show in the following that the use of two crossed linear arrays sometimes increases the complexity.

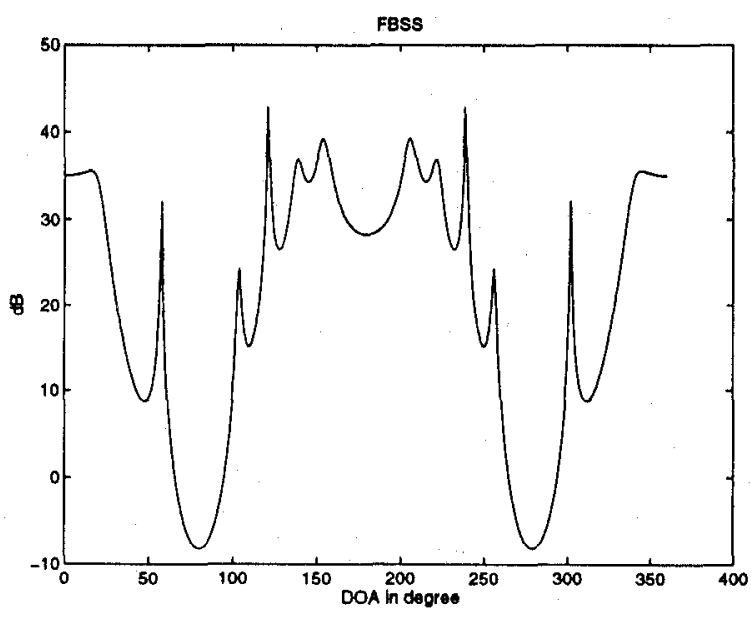

(a)
We used two crossed linear arrays each of nine sensors. We divided each linear array into two subarrays each of eight sensors. The spacing between two neighboring sensors is $0.45 \lambda$. We use these two crossed linear arrays to receive the same transmitted signals described in Example 1. Fig. 6(a) shows the DOA estimation using the horizontally positioned array. Two signals at $10^{\circ}$ and $20^{\circ}$ cannot be resolved. Fig. 6(b) shows the result of DOA estimation using the vertically positioned array. The final decisions on the DOAs are decided based upon the common peaks in these two figures. We need to search for peaks of DOAs twice.

EXAMPLE 3 We use a nine-sensor square array with a spacing of $0.45 \lambda$ between neighboring sensors to receive two coherent signals with DOAs at $75^{\circ}$ and $100^{\circ}$. The signal-to-noise ratio (SNR) is $20 \mathrm{~dB}$. A total of 500 samples are taken from the array each time. We apply SS and FBSS separately. Fig. 7 shows that the DOA estimation resolution achieved by a central symmetric array is significantly improved by using the FBSS method. The standard deviation is averaged over 200 estimated DOAs.

EXAMPLE 4 We use a nine-sensor square array to receive two coherent signals, one is at an azimuth of $40^{\circ}$ and an elevation of $30^{\circ}$, and the other is at an azimuth of $50^{\circ}$ and an elevation of $60^{\circ}$. The SNR is $20 \mathrm{~dB}$. There is a random phase delay from $(0,2 \pi)$ between these two signals at each snapshot. The number of samples taken is 500. By using FBSS and MUSIC, we obtain the result in Fig. 8. It demonstrates that a planar array enables us to perform FBSS and DOAs estimation in a 3D domain while a linear array or two crossed linear arrays are not capable of doing that.

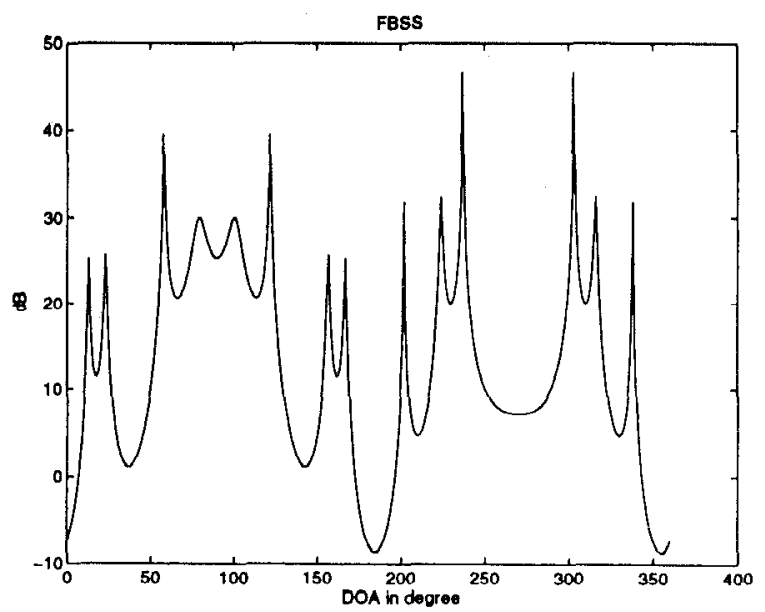

(b)

Fig. 6. DOA estimation of four groups of coherent signals at $\left(10^{\circ}, 20^{\circ}, 100^{\circ}\right),\left(120^{\circ}\right),\left(200^{\circ}, 220^{\circ}\right),\left(300^{\circ}\right)$ based on two acrossed linear arrays. (a) Horizontally positioned linear array. (b) Vertically positioned linear array. 


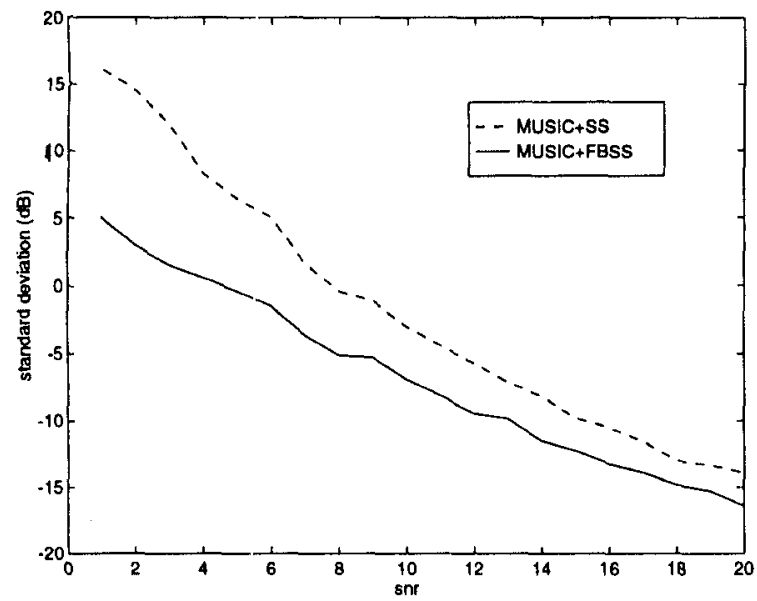

Fig. 7. Standard deviation of DOA estimation using SS and FBSS.

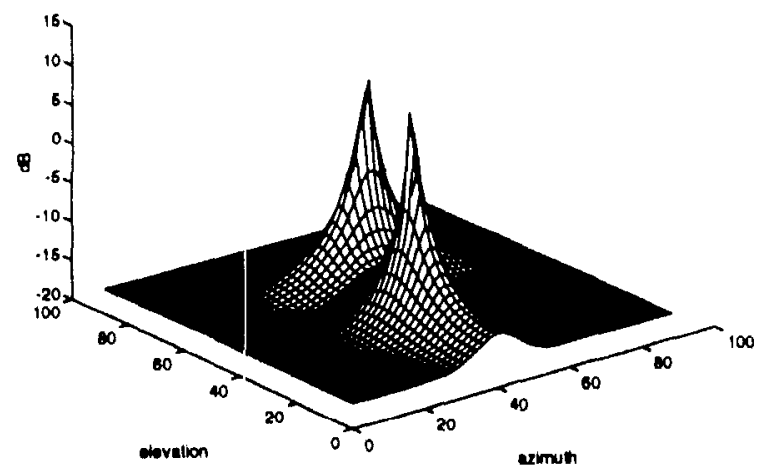

Fig. 8. DOA estimation of two coherent signals at azimuth of $40^{\circ}$ and elevation of $30^{\circ}$, and azimuth of $50^{\circ}$ and elevation of $60^{\circ}$, respectively.

\section{SS FOR ESPRIT}

Similar to MUSIC, the ESPRIT algorithm [8] is an approach to signal parameter estimation. It exploits an underlying data model at significant computational savings. The ESPRIT algorithm is also limited to estimating parameters in noncoherent incoming signals. The conventional SS can be incorporated into ESPRIT [30], but it requires the center array to be a uniformly spaced linear array. In this section, we show that our scheme also works for the ESPRIT algorithm to estimate parameters in a coherent interference environment.

In the ESPRIT algorithm, we consider $d$ narrowband plane; waves with incident angles $\theta_{1}, \ldots \theta_{d}$, and wavelength $\lambda$, impinge on a planar array of $m$ sensors ( $m$ is even), arranged in $m / 2$ doublet pairs. The displacement vector is the same for each doublet pair, but the location of each pair is arbitrary. The sensor output $x(t ;$ is given by

$$
\mathbf{x}(t)=\left(\begin{array}{c}
A \\
A \Phi
\end{array}\right) \mathbf{s}(t)+\mathbf{n}(t)
$$

where $\mathbf{n}(t)$ is a white Gaussian noise vector. $A$ and $A \Phi$ are the steering matrices corresponding to the first sensors and the second sensors in all pairs, respectively. The matrix $\Phi$ is a diagonal $d \times d$ matrix of phase delays between the doublet sensors for the $d$ signals. The sensor output covariance matrix $R_{x}$ is thus measured by

$$
R_{x}=\left(\begin{array}{c}
A \\
A \Phi
\end{array}\right) R_{s}\left(\begin{array}{c}
A \\
A \Phi
\end{array}\right)^{H}+\sigma^{2} I .
$$

A full rank matrix $R_{s}$ is assumed when the ESPRIT algorithm is performed. If some of the incoming signals are coherent, $R_{s}$ will not be a full rank matrix and the ESPRIT will fail. The SS technique we introduced in the previous sections can then be applied here to get a modified full rank signal covariance matrix.

We consider each doublet sensor pair in the array used by ESPRIT algorithm as one element. Then the array consists of $m / 2$ elements. If this array has an orientational invariance structure with $K$ subarrays and the corresponding center array has an ambiguity-free structure, the sensor output at the $k$ th subarray is given by

$$
\mathbf{x}_{k}(t)=\left(\begin{array}{c}
A_{1} \\
A_{1} \Phi
\end{array}\right) D_{k} \mathbf{s}(t)+\mathbf{n}(t) .
$$

Matrix $D_{k}$ is a diagonal $d \times d$ matrix of the phase delays in the form given in (13). The corresponding covariance matrix $R_{x_{k}}$ is given by

$$
R_{x_{k}}=\left(\begin{array}{c}
A_{1} \\
A_{1} \Phi
\end{array}\right) D_{k} R_{s} D_{k}^{H}\left(\begin{array}{c}
A_{1} \\
A_{1} \Phi
\end{array}\right)^{H}+\sigma^{2} I .
$$

A smoothed output covariance matrix $\bar{R}_{x}$ can thus be defined as

$$
\bar{R}_{x}=\frac{1}{K} \sum_{i=1}^{K} R_{x_{k}}=\left(\begin{array}{c}
A_{1} \\
A_{1} \Phi
\end{array}\right) \bar{R}_{s}\left(\begin{array}{c}
A_{1} \\
A_{1} \Phi
\end{array}\right)^{H}+\sigma^{2} I
$$

where $\bar{R}_{s}$ is the modified signal covariance matrix as defined in (16). As proved in Section II, $\bar{R}_{s}$ is of full rank if $K$ is larger than or equal to the size of the largest group of coherent signals. We can now successfully perform ESPRIT based on $\bar{R}_{x}$. We can also use FBSS to further reduce the number of sensors required and to improve the estimation resolution if the array of $m / 2$ element is central symmetric.

Although SS enables ESPRIT to estimate DOAs in a coherent interference environment, the estimation is still limited to identifying DOAs within $180^{\circ}$ in an azimuth-only system. Hence, in terms of performance robustness to DOAs, our SS is more effective for MUSIC than for ESPRIT.

EXAMPLE 5 A twelve-sensor array shown in Fig. 9 is used in this example to receive two coherent signals 


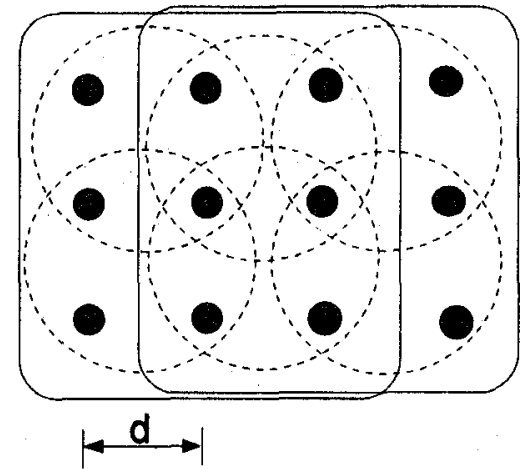

Fig. 9. Twelve-sensor rectangle array with spacing $d$.

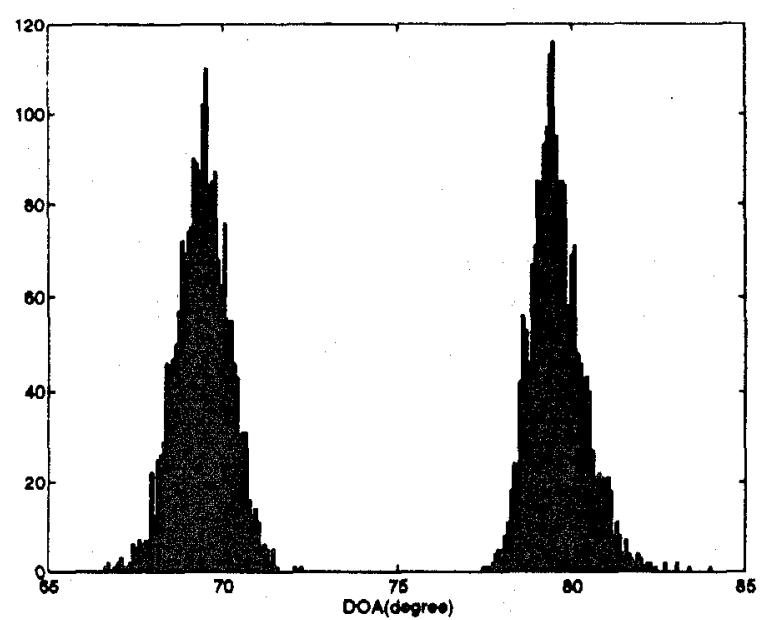

Fig. 10. FBSS and ESPRIT for DOA estimation of two coherent signals at $70^{\circ}$ and $80^{\circ}$.

at $70^{\circ}$ and $80^{\circ}$. This array consists of two overlapping nine-sensor square arrays. Each sensor in one square array and its counterpart in another form a doublet pair. These nine doublet pairs form an array which has orientational invariance structure and is central symmetric. The spacing between two neighboring sensors is $0.45 \lambda$. The doublet spacing for ESPRIR is $0.45 \lambda$. The SNR is $20 \mathrm{~dB}$. A total of 2000 trials are run. A histogram of the results is given in Fig. 10. We apply FBSS first and then apply the ESPRIT. The two angles are clearly identified.

\section{CONCLUSIONS}

To make constrained adaptive array beamforming and eigen-decomposition based DOA estimation algorithm effective in a coherent interference mobile channel environment, and to decorrelate coherent signals from arbitrary directions, we analyzed the SS on two-dimensional arrays. In order to apply SS to a two-dimensional array, this array must have an orientational invariance structure and its center array must be ambiguity free. Also the number of subarrays must be greater than or equal to the largest number of mutually coherent signals. To apply SS in conjunction with MUSIC, all the subarrays must also be ambiguity free, and the number of sensors in each subarrays must be larger than the number of incoming signals. For ESPRIT, two identical arrays (or subarrays) separated by a displacement vector are used each satisfying the conditions for applying SS and MUSIC.

When a nonlinear array is central symmetric, the FBSS can be used and it outperforms the regular SS in terms of improved efficiency and estimation resolution.

We proved the necessary and sufficient conditions for a three-sensor array manifold to be ambiguity free. We identified several situations, for higher order sensor array manifolds, in which ambiguity may arise. It is necessary to avoid the identified ambiguities in designing ambiguity free center arrays and subarrays.

\section{APPENDIX}

\section{PROOF OF LEMMA 1}

If part:

The proof is obvious and is omited.

\section{Only If part:}

If $B=A C, A=\left[a\left(\theta_{1}\right), \ldots, a\left(\theta_{d}\right)\right]$ and $B=$

$\left[b\left(\theta_{1}\right), \ldots, b\left(\theta_{d}\right)\right]$ and also assume $C$ is not a diagonal matrix, i.e., it has non-zero element $c_{l m}$ for $l \neq m$, then the steering vector $b\left(\theta_{m}\right)$ is

$$
b\left(\theta_{m}\right)=\sum_{i=1}^{d} c_{i m} a\left(\theta_{i}\right)=c_{l m} a\left(\theta_{l}\right)+\sum_{i=1, i \neq l}^{d} c_{i m} a\left(\theta_{i}\right) .
$$

This means that $b\left(\theta_{m}\right)$ is a function of variable $\theta_{l}$, which contradicts the definition that $b\left(\theta_{m}\right)$ is only a function of $\theta_{m}$. Thus the assumption that $C$ is a nondiagonal matrix is false. $C$ has to be a diagonal matrix with $c_{i i}=b\left(\theta_{i}\right) / a\left(\theta_{i}\right)$.

\section{PROOF OF LEMMA 2}

If part:

Obviously, $B$ can be any of $\left\{A_{1}, A_{2}, \ldots, A_{K}\right\}$.

\section{Only if part:}

If each $A_{i}$ can be mapped to a steering matrix $B$, by definition there exist $C_{i}, C_{j}$ such that $A_{i}=B C_{i}$, $A_{j}=B C_{j}$. By Lemma $1, C_{i}$ is a diagonal matrix. So $C_{i}^{-1}$ exists and is also a diagonal matrix. We have $A_{j}=A_{i} C_{i}^{-1} C_{j}$. Let $C_{i j}=C_{i}^{-1} C_{j}, C_{i j}$ is the product of two diagonal matrices. So $C_{i j}$ is also a diagonal matrix. $A_{j}=A_{i} C_{i j}$.

\section{PROOF OF THEOREM 3}

If part:

If sensors $A, B$, and $C$ are not on one line and their mutual distance is less than $\lambda / 2$, without loss of generality, we let sensor $\mathrm{A}$ be the first sensor in the array, $B$ the second, and $C$ the third. The steering 
matrix of the array has the form

$$
V=\left[\begin{array}{ccc}
1 & 1 & 1 \\
\exp \left(j \phi_{1}\left(\theta_{1}\right)\right) & \exp \left(j \phi_{1}\left(\theta_{2}\right)\right) & \exp \left(j \phi_{1}\left(\theta_{3}\right)\right) \\
\exp \left(j \phi_{2}\left(\theta_{1}\right)\right) & \exp \left(j \phi_{2}\left(\theta_{2}\right)\right) & \exp \left(j \phi_{2}\left(\theta_{3}\right)\right)
\end{array}\right]
$$

where $\phi$ denotes phase delay. If the distance between any two sensors is $<\lambda / 2$, the phase delay $\phi_{1}\left(\theta_{i}\right)$ and $\phi_{2}\left(\theta_{i}\right), i=1,2,3$, are real numbers from $(-\pi, \pi)$.

Note that the steering matrix of the array corresponding to three incoming signals at different angles is a special case of the general array in [26, Lemma 2]. By Lernma 2 in [26], $V$ is nonsingular with possible exception in one of the following three situations.

1) When $\phi_{1}\left(\theta_{1}\right)=\phi_{1}\left(\theta_{2}\right)$, i.e., the two incoming signals are symmetric with respect to the line on which sensors $A$ and $B$ are located. Note that

$$
\begin{aligned}
\operatorname{det}(V) & =\mid \begin{array}{c}
0 \\
\exp \left(j \phi_{1}\left(\theta_{1}\right)\right)-\exp \left(j \phi_{1}\left(\theta_{2}\right)\right) \\
\exp \left(j \phi_{2}\left(\theta_{1}\right)\right)-\exp \left(j \phi_{2}\left(\theta_{2}\right)\right)
\end{array} \\
& =-\mid \begin{array}{l}
\exp \left(j \phi_{1}\left(\theta_{1}\right)\right)-\exp \left(j \phi_{1}\left(\theta_{2}\right)\right) \\
\exp \left(j \phi_{2}\left(\theta_{1}\right)\right)-\exp \left(j \phi_{2}\left(\theta_{2}\right)\right)
\end{array}
\end{aligned}
$$

When $\phi_{1}\left(\theta_{1}\right)=\phi_{1}\left(\theta_{2}\right), \operatorname{det}(V)=0$ if and only if $\phi_{2}\left(\theta_{1}\right)=\phi_{2}\left(\theta_{2}\right)$ or $\phi_{1}\left(\theta_{3}\right)=\phi_{1}\left(\theta_{2}\right)$. Since these sensors are not on one line, if $\phi_{1}\left(\theta_{1}\right)=\phi_{1}\left(\theta_{2}\right)$, we have $\phi_{2}\left(\theta_{1}\right) \neq \phi_{2}\left(\theta_{2}\right)$. Since $\theta_{1}, \theta_{2}$ and $\theta_{3}$ are three different angles, when $\theta_{1}$ and $\theta_{2}$ are symmetric with respect to the line, $\theta_{3}$ and $\theta_{2}$ can not be symmetric to the line, i.e., if $\phi_{1}\left(\theta_{1}\right)=\phi_{1}\left(\theta_{2}\right)$, then we get $\phi_{1}\left(\theta_{3}\right) \neq$ $\phi_{1}\left(\theta_{2}\right)$. Thus, when $\phi_{1}\left(\theta_{1}\right)=\phi_{1}\left(\theta_{2}\right)$, the matrix $V$ is nonsingular.

2) Similarly, we can prove that when $\phi_{2}\left(\theta_{1}\right)=$ $\phi_{2}\left(\theta_{2}\right)$, the matrix $V$ is nonsingular.

3) When $\phi_{1}\left(\theta_{1}\right)-\phi_{1}\left(\theta_{2}\right)=\phi_{2}\left(\theta_{1}\right)-\phi_{2}\left(\theta_{2}\right)$, i.e. $\phi_{1}\left(\theta_{1}\right)-\phi_{2}\left(\theta_{1}\right)=\phi_{1}\left(\theta_{2}\right)-\phi_{2}\left(\theta_{2}\right), \theta_{1}$ and $\theta_{2}$ are symmetric with respect to the line connecting sensors $B$ and $C$. Note that
When $\phi_{1}\left(\theta_{1}\right)-\phi_{2}\left(\theta_{1}\right)=\phi_{1}\left(\theta_{2}\right)-\phi_{2}\left(\theta_{2}\right), \operatorname{det}(V)=0$ if and only if $\exp \left(-j \phi_{2}\left(\theta_{1}\right)\right)=\exp \left(-j \phi_{2}\left(\theta_{2}\right)\right)$ or $\exp \left(j \phi_{1}\left(\theta_{2}\right)-j \phi_{2}\left(\theta_{2}\right)\right)=\exp \left(j \phi_{1}\left(\theta_{3}\right)-j \phi_{2}\left(\theta_{3}\right)\right)$. Since the mutual distance between $A, B$, and $C$ are less than $\lambda / 2, \phi_{2}\left(\theta_{1}\right), \phi_{2}\left(\theta_{2}\right), \phi_{1}\left(\theta_{2}\right)-\phi_{2}\left(\theta_{2}\right)$ and $\phi_{1}\left(\theta_{3}\right)-\phi_{2}\left(\theta_{3}\right)$ are all real numbers in $(-\pi, \pi)$. $\exp \left(-j \phi_{2}\left(\theta_{1}\right)\right)=\exp \left(-j \phi_{2}\left(\theta_{2}\right)\right)$ if and only if $\phi_{2}\left(\theta_{1}\right)=$ $\phi_{2}\left(\theta_{2}\right) \cdot \exp \left(j \phi_{1}\left(\theta_{2}\right)-j \phi_{2}\left(\theta_{2}\right)\right)=\exp \left(j \phi_{1}\left(\theta_{3}\right)-j \phi_{2}\left(\theta_{3}\right)\right)$ if and only if $\phi_{1}\left(\theta_{2}\right)-\phi_{2}\left(\theta_{2}\right)=\phi_{1}\left(\theta_{3}\right)-\phi_{2}\left(\theta_{3}\right)$.

Since $A, B$, and $C$ are not on one line, if $\theta_{1}$ and $\theta_{2}$ are symmetric to the line connecting $B$ and $C$, they cannot be symmetric to the line connecting $A$ and $B$ or $A$ and $C$. That is, if $\phi_{1}\left(\theta_{1}\right)-\phi_{2}\left(\theta_{1}\right)=\phi_{1}\left(\theta_{2}\right)-\phi_{2}\left(\theta_{2}\right)$, we have $\phi_{2}\left(\theta_{1}\right) \neq \phi_{2}\left(\theta_{2}\right)$. Since $\theta_{1}, \theta_{2}$, and $\theta_{3}$ are three different incoming angles, if $\theta_{1}$ and $\theta_{2}$ are symmetric to the line connecting $B$ and $C, \theta_{2}$ and $\theta_{3}$ cannot be symmetric to the line. That is, if $\phi_{1}\left(\theta_{1}\right)-\phi_{2}\left(\theta_{1}\right)=$ $\phi_{1}\left(\theta_{2}\right)-\phi_{2}\left(\theta_{2}\right)$, we have $\phi_{1}\left(\theta_{2}\right)-\phi_{2}\left(\theta_{2}\right) \neq \phi_{1}\left(\theta_{3}\right)-$

$$
\begin{aligned}
& \exp \left(j \phi_{1}\left(\theta_{3}\right)\right)-\exp \left(j \phi_{1}\left(\theta_{2}\right)\right) \\
& \exp \left(j \phi_{2}\left(\theta_{3}\right)\right)-\exp \left(j \phi_{2}\left(\theta_{2}\right)\right)
\end{aligned}
$$

$\phi_{2}\left(\theta_{3}\right)$. Thus, when $\phi_{1}\left(\theta_{1}\right)-\phi_{2}\left(\theta_{1}\right)=\phi_{1}\left(\theta_{2}\right)-\phi_{2}\left(\theta_{2}\right)$, the matrix $V$ is nonsingular.

Therefore, we conclude that all the three situations which cause the singularity of the matrix in [26, Lemma 2] will not cause the singularity of three-sensor steering matrix if three sensors are not on one line and their mutual distance is less than $\lambda / 2$. Therefore the matrix $V$ is full rank.

If the spacing between any two of the three sensors is not larger than $\lambda / 2$, and there is at least one pair in these three sensors with a spacing of $\lambda / 2$, then the only situation that the phase delay $\phi_{1}\left(\theta_{i}\right)$ and $\phi_{2}\left(\theta_{i}\right)$, $i=1,2,3$, are not all in $(-\pi, \pi)$ is when one of the incoming signals is from the direction parallel to a line on which the two sensors with spacing $\lambda / 2$ are

$$
\begin{aligned}
\operatorname{det}(V) & =\left|\begin{array}{ccc}
\exp \left(-j \phi_{2}\left(\theta_{1}\right)\right) & \exp \left(-j \phi_{2}\left(\theta_{2}\right)\right) & \exp \left(-j \phi_{2}\left(\theta_{3}\right)\right) \\
\exp \left(j \phi_{1}\left(\theta_{1}\right)-j \phi_{2}\left(\theta_{1}\right)\right) & \exp \left(j \phi_{1}\left(\theta_{2}\right)-j \phi_{2}\left(\theta_{2}\right)\right) & \exp \left(j \phi_{1}\left(\theta_{3}\right)-j \phi_{2}\left(\theta_{3}\right)\right) \\
1 & 1
\end{array}\right| \\
& =\left(\exp \left(-j \phi_{2}\left(\theta_{1}\right)\right)-\exp \left(-j \phi_{2}\left(\theta_{2}\right)\right)\right)\left(\exp \left(j \phi_{1}\left(\theta_{2}\right)-j \phi_{2}\left(\theta_{2}\right)\right)-\exp \left(j \phi_{1}\left(\theta_{3}\right)-j \phi_{2}\left(\theta_{3}\right)\right)\right) .
\end{aligned}
$$


located. The other two signals can be either from the opposite direction or from other directions. If one of the other two signals is from the opposite direction, it can be easily proved that the corresponding steering matrix is full rank. If the other two signals are from the two other different directions, then one of $\phi_{n}\left(\theta_{i}\right)$, $n=1,2, i=1,2,3$ is equal to $\pi$ and the rest are real numbers from $(-\pi, \pi)$. Similarly, we can prove that the matrix $V$ is of full rank.

\section{Only if part:}

If the conditions in Theorem 1 are not satisfied, rank- 1 or rank- 2 ambiguity occurs for some incoming signals. These situations are shown schematically in Fig. 2(a) and (b). In Fig. 2(a), the relation between $\theta$ and $\alpha$ is

$$
\begin{array}{r}
2 \pi \frac{d}{\lambda} \sin (\theta-\alpha)+k 2 \pi=2 \pi \frac{d}{\lambda} \sin (\theta+\alpha) \\
k \in\{1,2, \ldots\} .
\end{array}
$$

In Fig. 2(b), the relation between $\theta$ and $\alpha$ is

$$
\begin{array}{r}
2 \pi \frac{d}{\lambda} \sin (\alpha)+k 2 \pi=2 \pi \frac{d}{\lambda} \sin \left(\frac{\pi}{2}-\theta\right), \\
k \in\{1,2, \ldots\} .
\end{array}
$$

\section{REFERENCES}

[1] Swales, S. C., Beach, M. A., Edwards, D. J., and McGeehan, J. P. (1990)

The performance enhancement of multibeam adaptive base-station antennas for cellular land mobile radio systems.

IEEE Transactions on Vehicular Technology, 39, 1 (Feb. 1990), 56-67.

[2] Anderson, S., Millnert, M., Viberg, M., and Wahlberg, B. (1991)

An adaptive array for mobile communication systems. IEEE Transactions on Vehicular Technology, 40, 1 (Feb 1991), 230-236.

[3] Lee, W. C. Y. (1994)

Applying the intelligent cell concept to PCS.

IEEE Transactions on Vehicular Technology, 43, 3 (Aug. 1994), 672-679.

[4] Winters, J. H. (1993)

Signal acquisition and tracking with adaptive arrays in the digital mobile radio system IS-54 with flat fading. IEEE Transactions on Vehicular Technology, 42, 4 (Nov. 1993), 377-384.

[5] Winters, J. H., Salz, J., and Gitlin, R. D. (1994)

The impact of antenna diversity on the capacity of wireless communication systems.

IEEE Transactions on Communications, 42, 2/3/4/

(Feb./Mar./Apr. 1994), 1740-1751.

[6] Brester, Y., and Macovski, A. (1986)

Exact maximum likelihood parameter estimation of superimposed exponential signals in noise.

IEEE Transactions on Acoustics, Speech, Signal Processing, ASSP-34 (Oct. 1986), 1081-1089.

[7] Schmidt, R. O. (1981)

A signal subspace approach to multiple source location and spectral estimation.

Ph.D. dissertation, Stanford University, Stanford, CA, May 1981.
[8] Roy, R., and Kailath, T. (1989)

ESPRIT-estimation of signal parameters via rotational invariance techniques.

IEEE Transactions on Acoustics, Speech, Signal Processing, 37, 7 (July 1989), 984-995.

[9] Capon, J. (1969)

High-resolution frequency-wavenumber spectrum analysis.

Proceedings of IEEE, 57 (Aug. 1969), 1408-1418.

[10] Mcwhirter, J. G., and Shepherd, T. J. (1989) Systolic array processor for MVDR beamforming. IEE Proceedings, Pt. F (London), 136 (Apr. 1989), 75-80.

[11] Frost, O. L. (1972)

An algorithm for linearly constrained adaptive array processing.

Proceedings of IEEE, 60 (Aug. 1972), 926-935.

[12] Shan, T. J., Wax, M., and Kailath, T. (1985)

On spatial smoothing for direction-of-arrival estimation of coherent signals.

IEEE Transactions on Acoustics, Speech, Signal Processing, ASSP-33, 4 (Aug. 1985), 806-811.

[13] Widrow, B., Duval, K. M., Gooch, R. P., and William, W. C. (1982)

Signal cancellation phenomena in adaptive antennas:

Causes and cures.

IEEE Transactions on Acoustics, Speech, Signal Processing, AP-30, 3 (May 1982), 469-478.

[14] Evans, J. E., Johnson, J. R., and Sun, D. F. (1981)

High resolution angular spectrum estimation techniques

for terrain scattering analysis and angle of arrival estimation.

In 1st ASSP Workshop Spectral Estimation, Hamilton, Canada, 1981.

[15] Shan, T. J., and Kailath, T. (1985)

Adaptive beamforming for coherent signals and interference.

IEEE Transactions on Acoustics, Speech, Signal Processing, 33, 3 (June 1985), 527-536.

[16] Reddy, V. U., Paulraj, A., and Kailath, T. (1987)

Performance analysis of the optimum beamformer in the presence of correlated sources and its behavior under spatial smoothing.

IEEE Transactions on Signal Processing, ASSP-35, 7 (July 1987), 927-936.

[17] Cadzow, J. A. (1988)

A high resolution direction-of-arrival algorithm for narrow-band coherent and incoherent sources. IEEE Transactions on Acoustics, Speech, Signal Processing, 36 (July 1988), 965-979.

[18] Viberg, M., and Ottersten, B. (1991)

Sensor array processing based on subspace fitting. IEEE Transactions on Signal Processing, 39, 5 (May 1991), 1110-1121.

[19] Viberg, M., Ottersten, B., and Kailath, T. (1991)

Detection and estimation in sensor arrays using weighted subspace fitting.

IEEE Transactions on Signal Processing, 39, 11 (Nov. 1991), 2436-2449.

[20] Friedlander, B., and Weiss, A. J. (1992)

Direction finding using spatial smoothing with interpolated arrays.

IEEE Transactions on Aerospace and Electronic System, 28, 2 (Apr, 1992), 574-586.

[21] Weiss, A. J., and Friedlander, B. (1993)

Performance analysis of spatial smoothing with interpolated arrays.

IEEE Transactions on Signal Processing, 41, 5 (May 1993), 1881-1892. 
[22] Haykin, S. (199:)

Adaptive Filter Theorem (2nd ed.).

Englewood Cliffs, NJ: Prentice-Hall (Information and

System Sciences Series), 1991.

[23] Pillai, S. U., and Kwon, B. H. (1989)

Forward/backward spatial smoothing techniques for coherent signal identification.

IEEE Transactions on Acoustics, Speech, Signal Processing,

37, 1 (Jan. 1989), 8-15.

[24] Proukakis, C., and Manikas, A. (1994)

Study of ambiguities of linear arrays.

In Proceedings of the International Conference on

Acoustics, Speech, Signal Processing, (1994), IV-549-552.

[25] Tan, K., and Goh, Z. (1994)

A construction of arrays free of rank ambiguities.

In Proceedings of the International Conference on

Acoustics, Speech, Signal Processing, (1994), IV-545-548.

[26] Lo, J. T.-H., and Marple, S. L. (1992)

Observability conditions for multiple signal direction

finding and array sensor localization.

IEEE Transactions on Signal Processing, 40, 11 (Nov.

1992), 2641-2650.

[27] Li, J. (1992)

Improved angular resolution for spatial smoothing

techniques.

IEEE Transactions on Signal Processing, 40 (Dec. 1992),

3078-3081.
[28] Shan, T., Paulraj, A., and Kailath, T. (1987)

On smoothed rank profile tests in eigenstructure methods for directions-of-arrival estimation.

IEEE Transactions on Acoustic, Speech, and Signal

Processing, ASSP-35 (Oct. 1987), 1377-1385.

[29] Litva, J., and Zeytinoghi, M. (1990)

Application of high-resolution direction-finding

algorithms to circular arrays with mutual coupling present.

Final report prepared for DREO under terms of Contract

W7714-9-9132/01-SZ, July 1990.

[30] Swindlchurst, A. L., Ottersten, B., Roy, R., and Kailath, T. (1992)

Multiple invariance ESPRIT.

IEEE Transactions on Acoustics, Speech, Signal Processing, 40, 4 (Apr. 1992), 867-881.

[31] Lee, W. C. Y. (1993)

Mobile Communications Design Fundamentals (2nd ed.). New York: Wiley, 1993.

[32] Cooper, M., and Roy, R. (1992)

SDMA technology-overview and development status. ArrayComm-ID-010, ArrayComm, Inc., CA, Sept. 1992.

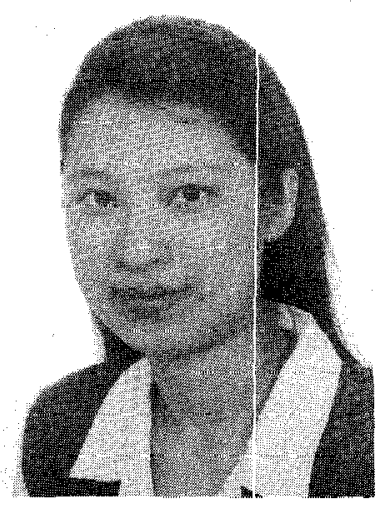

Hongyi Wang received the B.S. degree in electrical engineering from Shanghai Jiao Tong University, China in 1985, the M.S. degrees in mathematical statistics and in electrical and computer engineering from the University of Massachusetts at Amherst in 1989 and 1992, respectively, and a Ph.D. degree in electrical engineering at the University of Maryland, College Park, in 1996.

From Jan. to Apr. 1995, she was a consultant to Digital Communications Research Department at AT\&T Bell Laboratories, where she was involved in the smart antenna design project. From June to Aug. 1996, she was with Telogy Networks Inc., where she worked on digital signal processing software design for CDMA base station testing. Since Sept. 1996, she has been a member of technical staff in Lucent Technologies, where she is working on lab simulation and performance analysis of IS-95 CDMA mobile communication systems. Her research interests include wireless communication systems performance analysis, RF channel simulation and modeling, and digital signal processing.



K. J. Ray Liu (S'86-M'90-SM'93) received the B.S. degree from the National Taiwan University in 1983, and the Ph.D. degree from the University of California, Los Angeles, in 1990 , both in electrical engineering.

Since 1990 he has been with the Electrical Engineering Department and Institute for Systems Research of University of Maryland at College Park, where he is an Associate Professor. During his sabbatical leave in 1996-1997, he was Visiting Associate Professor at Stanford University and Chief Scientist of NeoParadigm Labs. His research interests span all aspects of signal processing with application to image and video, wireless communications, networking, and medical and biomedical technology.

Dr. Liu received numerous awards including the 1994 National Science Foundation Young Investigator Award, the IEEE Signal Processing Society's 1993 Senior Award (Best Paper Award), the George Corcoran Award in 1994 for outstanding contributions to electrical engineering education and the 1995-96 Outstanding Systems Engineering Faculty Award in recognition of outstanding contributions in interdisciplinary research, both from the University of Maryland, and many others. Dr. Liu is an Associate Editor of IEEE Transactions on Signal Processing, a Guest Editor of special issues on Multimedia Signal Processing and Technology of Proceedings of the IEEE, a Guest Editor of special issues on Signal Processing for Wireless Communications of IEEE Journal of Selected Areas in Communications, an editor of Journal of VLSI Signal Processing, a member of Design and Implementation of Signal Processing Systems Technical Committee, and a founding member of Multimedia Signal Processing Technical Committee of IEEE Signal Processing Society. 